\title{
Encapsulation of Berberine in Nano-Sized PLGA Synthesized by Emulsification Method
}

\author{
Manisha Khemani, ${ }^{1}$ Maheshwar Sharon, ${ }^{2}$ and Madhuri Sharon ${ }^{2}$ \\ ${ }^{1}$ Department of Chemistry, Momin College, Maharashtra, Bhiwandi, India \\ ${ }^{2}$ N.S.N. Research Centre for Nanotechnology \& Bionanotechnology, Jambhul Phata, Maharashtra, Ambernath 421 505, India
}

Correspondence should be addressed to Madhuri Sharon, sharonmadhuri@yahoo.com

Received 30 April 2012; Accepted 17 July 2012

Academic Editors: Y. I. Jeong, C. Li, and P. Perriat

Copyright (C) 2012 Manisha Khemani et al. This is an open access article distributed under the Creative Commons Attribution License, which permits unrestricted use, distribution, and reproduction in any medium, provided the original work is properly cited.

Nanoparticles of PLGA (polylactide glycolic acid) were prepared using biodegradable poly (D, L-lactide-co-glycolide)—75:25, by emulsification method using PVA (Mol. Wt. 9000) or didodecyl dimethyl ammonium bromide (DMAB) as surfactant. Nanoparticles were morphologically characterized using scanning electron microscope (SEM) and particle size analyzer. The distribution of size of PLGA nanoparticles was in the range of $48-211 \mathrm{~nm}$. Berberine, a yellow isoquinoline alkaloid that is used as traditional anticancer drug, was loaded on to PLGA nanoparticles by single emulsion as well as multiple emulsion solvent evaporation techniques. Particle size analysis showed an increase in berberine loaded PLGA NP size to 180-310 nm when PVA was used as a stabilizer. Whereas use of DMAB as a stabilizer led to precipitation. In vitro drug release analysis revealed that acidic $\mathrm{pH}$ of 5.5 was more suitable for release of berberine than $\mathrm{pH}$ 7.4.

\section{Introduction}

Nanometals as well as organic nanoparticles (NP) as drug delivery devices is rapidly expanding area in drug delivery sciences. Organic polymer like polylactide glycolic acid (PLGA) has been used earlier as drug carrier because it is biocompatible as well as biodegradable $[1,2]$. However, nowaday's approaches are to use nano-sized PLGA as drug delivery vehicle. Tremendous opportunities exist for using nanoparticles as controlled drug delivery systems especially for cancer therapy so as to expose the patients with reduced doses [3-5].

Berberine, a naturally occurring isoquinoline alkaloid, is present in the roots, rhizome, and stem bark of a number of medicinal plants. Berberine has tremendous potential to cure many physiological disorders; hence, it has been used in the Ayurvedic [6], Unani, and Chinese as well as Homeopathic medicine. Berberine has been shown to inhibit activator protein 1, a key transcription factor in inflammation and carcinogenesis, in human cell lines [7] and has been shown to possess antitumor properties and effectively inhibit cyclooxygenase-2 transcriptional activity in human colon cancer cells $[8,9]$. Berberine is known to inhibit DNA topoisomerase II [10]. Moreover, the antitumor properties of berberine are now recognized by researchers and clinical oncologists. The effects of berberine on human malignant brain tumor, esophageal cancer, and human leukemic and human colon cancer cell lines have been tested and significant killing effects have been achieved $[1,2,11]$. After looking into all these properties of Berberine, it was decided to try to encapsulate it in NP of PLGA for facilitated drug delivery (Scheme 1).

\section{Materials and Methods}

PLGA $(75: 25)$ was provided as gift by Purasorb. Berberine chloride was purchased from Sigma Aldrich (Bangalore, India). Poly vinyl alcohol (PVA; Mol. Wt. 9000) or Didodecyl 


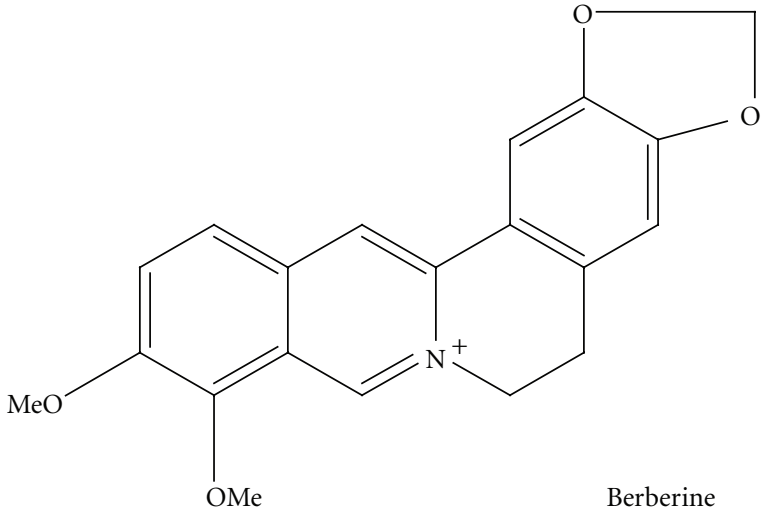

Scheme 1: Berberine.

dimethyl ammonium bromide (DMAB) was used as surfactant.

2.1. Synthesis of Poly (D, L-lactide-co-glycolide)-75:25 (PLGA) Nanoparticles (NP). PLGA, a biodegradable polymer, NPs were prepared by emulsification diffusion solvent evaporation method. $50 \mathrm{mg}$ of PLGA was dissolved in $5 \mathrm{~mL}$ of various organic solvents like ethyl acetate (EA), acetone + ethanol (ACE), dichloro-methane (DCM) or mixture of ACE and DCM. This oil phase was added to $5 \mathrm{~mL}$ water containing PVA (of various Mol. Wt, as stabilizer). This mixture was homogenized at 15,000 rpm for $3 \mathrm{~min}$ then $50 \mathrm{~mL}$ of distilled water was added to solidify the NPs. The organic solvent was evaporated by stirring the solution for $8 \mathrm{hrs}$ at $40^{\circ} \mathrm{C}$. The NPs were collected by centrifugation at $16,000 \mathrm{rpm}$ and washed with D.W and stored in refrigerator.

2.2. Loading Berberine onto PLGA Nanoparticles. It was done by single emulsion as well as double emulsion method. $50 \mathrm{mg}$ of PLGA was dissolved in $2 \mathrm{~mL}$ of organic phaseconsisting of either DCM or acetone or the mixture of both.

In single emulsion method berberine, dissolved in methanol, was added to this organic phase and then it was emulsified with $15 \mathrm{~mL}$ aqueous phase containing 1\% PVA (M0l. Wt 9000) at $15000 \mathrm{rpm}$ using high speed homogenizer for $8 \mathrm{~min}$. The organic solvent was evaporated by stirring.

In the multiple emulsion method the aqueous solution of berberine was first emulsified with organic phase consisting of either DCM or acetone or the mixture of both and then it was emulsified with $30 \mathrm{~mL}$ of aqueous phase containing $1 \%$ PVA. The residual organic solvent was evaporated by stirring for 5 hrs. The NPs suspended in emulsion were collected by ultracentrifugation at $16,000 \mathrm{rpm}$ and washed thrice with distilled water. Finally the particles were dried and stored in refrigerator, till further use

2.3. Assessment of Particle Size and Size Distribution. Particle size analysis was done by a dynamic light scattering (DLS) method using Zetasizer Nano ZS (Malven Instruments, UK).
2.4. Surface Morphology of PLGA-Berberine Nanoparticles. PLGA morphology was assessed by scanning electron microscopy (SEM). SEM was performed on a Carl Zeiss Microimaging, GmbH, Germany. For sample preparation, 23 drops of the colloidal gold solution were dispensed onto a silicon wafer and dried under ambient condition before examination. Particles were mounted on a metal hub using carbon adhesive tape and coated with a mixture of gold and palladium in an argon atmosphere.

2.5. Determination of Drug Loading and Encapsulation. The berberine content of nanoparticles was determined by a spectrophotometer (Perkin-Elmer). The fluorescence intensity of free Berberine in centrifugate was measured at $420 \mathrm{~nm}$. The concentration of berberine was calculated from a standard curve, prepared by measuring the fluorescence intensity of known concentration of free berberine. The percentage drug entrapment was calculated as follows:

Percentage drug entrapment

$$
=\frac{(\text { Mass of total drug }- \text { Mass of free drug }) * 100}{\text { Mass of total drug }} .
$$

2.6. Confirmation of Berberine Loading by FTIR. FTIR analysis of PLGA, berberine and berberine entrapped PLGA was done by FT/IR-4100 type A.

2.7. In Vitro Berberine Release Study. In vitro release studies of berberine, loaded on to PLGA NPs were carried out in phosphate buffer solution (PBS) at $\mathrm{pH} 7.4$ and $\mathrm{pH}$ 5.5. The NPs were dispersed in $1 \mathrm{~mL}$ of PBS in a dialysis bag, and it was suspended in $50 \mathrm{~mL}$ of PBS and placed in incubator at $37^{\circ} \mathrm{C}$. At regular intervals the absorbance was checked at $420 \mathrm{~nm}$ for the amount of drug released.

\section{Results and Discussions}

As mentioned above the PLGA NPs were prepared by emulsification-diffusion solvent evaporation method using organic solvents like EA, ACE, and DCM. The stabilizers used were PVA, didodecyl dimethyl ammonium bromide (DMAB), and Sodium dodecyl sulphate (SDS). During the process organic solvent diffuses to the external phase leading to the formation of NPs. The size of NPs was found to be dependent on a number of parameters like the type of surfactant, concentration of surfactant (Table 1) and type of organic solvent (Table 2) used for synthesis.

3.1. Impact of Surfactant on the Particle Size of PLGA. Table 1 and Figure 1 show distributions of the particle size of PLGA nanoparticles prepared with different surfactants. Particles of smallest size about $50 \mathrm{~nm}$ were obtained with $0.5 \%$ DMAB in EA solvent. The particle size was found to increase with the increase in concentration of DMAB, that is, up to $102 \mathrm{~nm}$ with $1 \%$ DMAB. 
TABLE 1: Effect of different surfactants and their Mol. Wt. on the size of PLGA nanoparticles synthesized by EDSE method using ethyl acetate as organic solvent.

\begin{tabular}{lcccc}
\hline S. N. & Surfactant used for PLGA synthesis & Mol. Wt. & Conc. of surfactant & Mean particle size \\
\hline 1 & Poly Vinyl Alcohol (PVA) & 115,000 & $1 \%$ & $312 \mathrm{~nm}$ \\
2 & PVA & 9,000 & $1 \%$ & $250 \mathrm{~nm}$ \\
3 & PVA & 9000 & $0.5 \%$ & $258 \mathrm{~nm}$ \\
4 & DMAB & 322 & $1 \%$ & $102 \mathrm{~nm}$ \\
5 & DMAB & 322 & $0.5 \%$ & $49 \mathrm{~nm}$ \\
6 & SDS & 288.38 & $1 \%$ & $214 \mathrm{~nm}$ \\
\hline
\end{tabular}

TABLE 2: Effect of solvent and 1\% PVA (as stabilizer) on size of PLGA nanoparticles.

\begin{tabular}{lcc}
\hline Sr. no. & Solvent & Particle size \\
\hline 1 & Acetone & $337 \mathrm{~nm}$ \\
2 & Ethyl acetate & $250 \mathrm{~nm}$ \\
3 & DCM & $211 \mathrm{~nm}$ \\
\hline
\end{tabular}

Similarly, use of PVA of low Mol. Wt. (9000) produced smaller NPs, that is, $48 \mathrm{~nm}$ (Figure 4) however some particles were as big as $250 \mathrm{~nm}$, as observed in particle size analysis data. PVA of higher Mol. Wt. $(1,15,000)$ produced particles up to $312 \mathrm{~nm}$. There was slight decrease in the particle size when the concentration of the PVA was increased to $1 \%$ which may be due to increase in the viscosity of the medium.

Sodium dodecyl sulphate (SDS) was another surfactant used for preparing PLGA NPs, and it produced NPs of size $214 \mathrm{~nm}$ but the yield of NPs was very less as compared to PVA.

The graphs show narrow particle size distribution in case of PVA (Mol. Wt. 9000) and DMAB. Also the results indicate that use of DMAB leads to formation of nanoparticles of smaller size ranging from $47 \mathrm{~nm}-98 \mathrm{~nm}$.

3.2. Impact of Solvent on the Particle Size of PLGA. As it can be seen in Table 2 and Figure 2, the size of NPs was also affected by different solvents and the concentration of surfactant used along with it.

Water miscible solvent acetone produced the particles of larger size whereas partially water miscible and water immiscible solvents produced particles of smaller size. The stabilizer remains adequately protected at the interface of two immiscible liquids (EA and DCM), leading to the formation of particles of smaller size.

In case of water miscible solvent like acetone, no proper emulsion is formed even in presence of stabilizer, hence it resulted in precipitation leading to the formation of larger particles [12]. They have also reported synthesis of 250$400 \mathrm{~nm}$ PLGA with the use of acetone and $350-450 \mathrm{~nm}$ with DCM.

In the present work, PLGA NPs of smaller size that is, $211 \mathrm{~nm}$ was produced when DCM along with very small quantity of acetone was used, whereas addition of larger quantities of acetone resulted in precipitation.
It is reported [13] that increased stability of nanoparticles with increase in concentration of PVA (nonionic stabilizer) which is due to the increased adsorption of stabilizer molecules on the surface of emulsion droplets thereby reducing the tendency of precipitation and hence smaller particles are formed. However, in case of DMAB the particle size increases with increase in concentration because it is a cationic stabilizer and has more tendencies to remain in the aqueous phase and does not play a significant role in emulsification.

Berberine loaded PLGA NPs - that were prepared by w/o emulsion and w/o/w emulsion method exhibited very significant impact of $\mathrm{pH}$ and surfactant.

3.3. Effect of $p H$ on Encapsulation Efficiency. The drug loading percentage at $\mathrm{pH} 7$ was calculated and it is presented in Table 3. The result obtained using w/o/w was repeated at $\mathrm{pH} 8$ and the results are presented in Table 4.

The results indicate that when DMAB was used as surfactant it initially leads to formation of PLGA NPs, which precipitated within few minutes resulting in formation of very large (average particle size- $1214 \mathrm{~nm}$ ) particles. As it can be seen in Figure 3(a) there are three separate peaks where the largest peak indicates aggregation of nanoparticles. The samples prepared using higher concentration of DMAB, resulted in faster precipitation (data not presented).

Hence, further samples were prepared using PVA. It can be seen that water immiscible solvent like DCM produces smallest particles and maximum entrapment whereas water miscible solvent like acetone produced larger particles and low encapsulation.

When the mixture of acetone and DCM was used, w/o/w method yielded particles of intermediate size and more entrapment. Whereas, w/o method yielded smaller particles and less entrapment.

When w/o/w method was tried with pure acetone and pure DCM, no encapsulation was observed. The poor encapsulation of berberine on PLGA can be attributed to its structure which does not have any potential functional group for attachment to polymer.

The encapsulation efficiency of the NPs was greatly affected by the $\mathrm{pH}$ of the solution. The double emulsion method which resulted in $40 \%$ encapsulation with DCM plus acetone at $\mathrm{pH} 7$ was enhanced to $58 \%$ encapsulation efficiency at $\mathrm{pH} 8$ under the same experimental conditions. This may be due to the reduced solubility of the alkaloid 


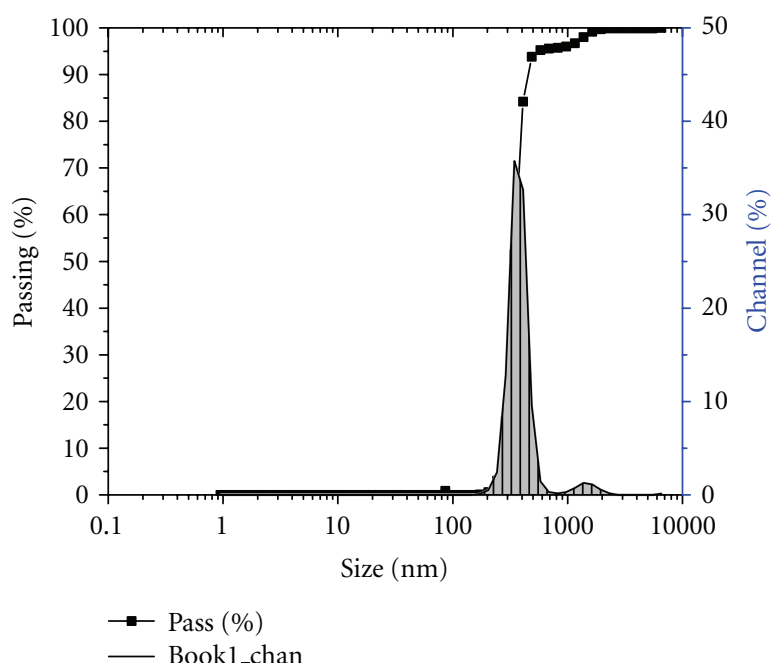

(a)

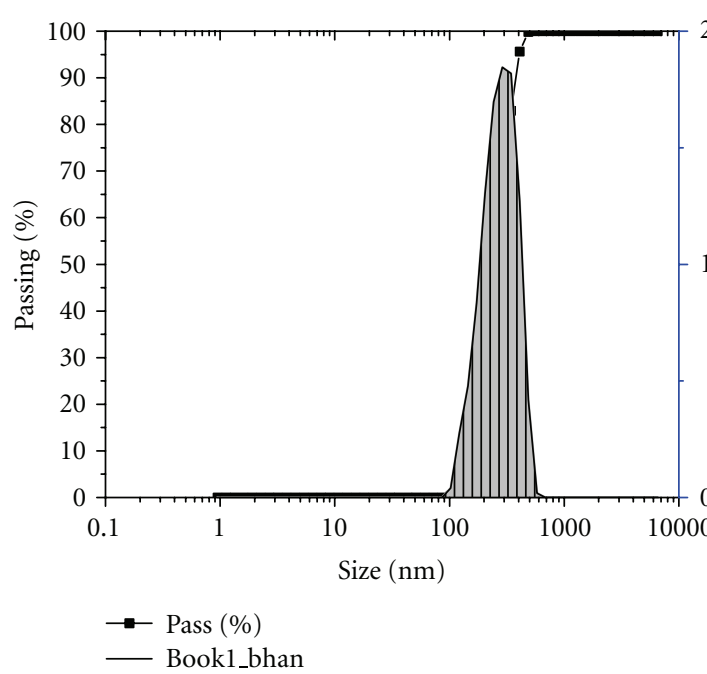

(c)

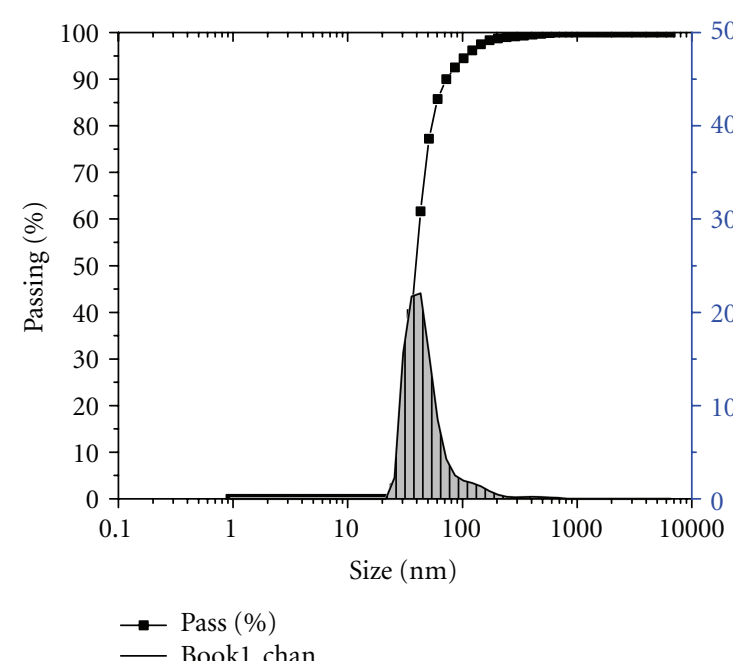

(e)

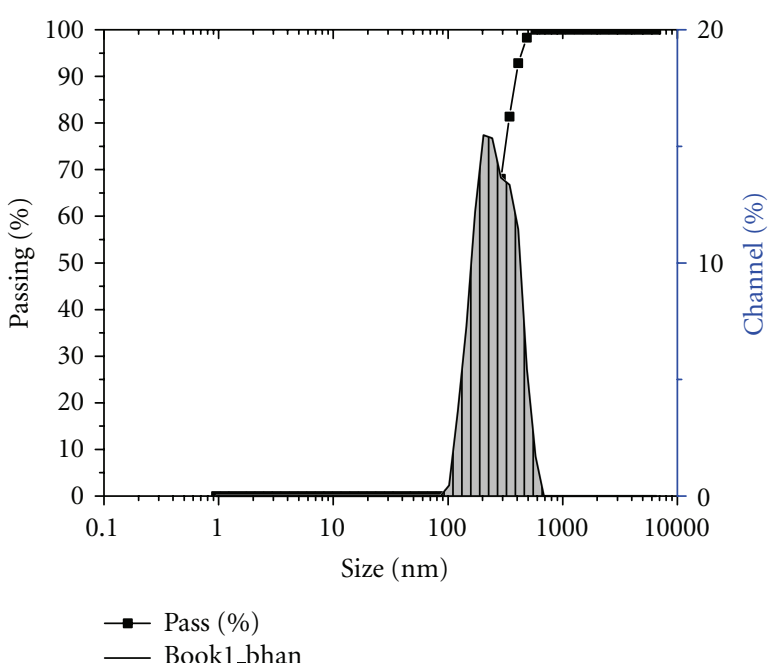

(b)

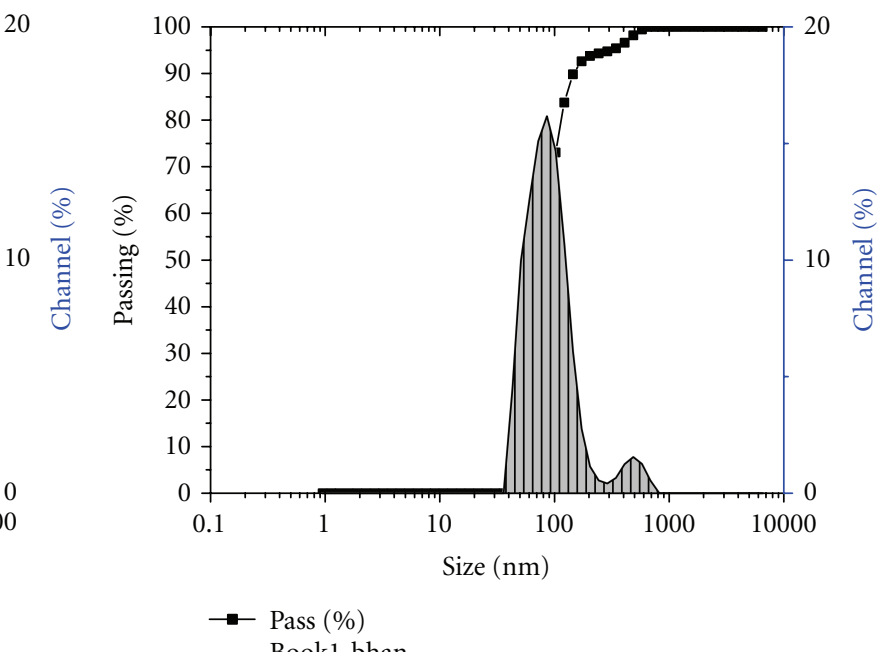

(d)

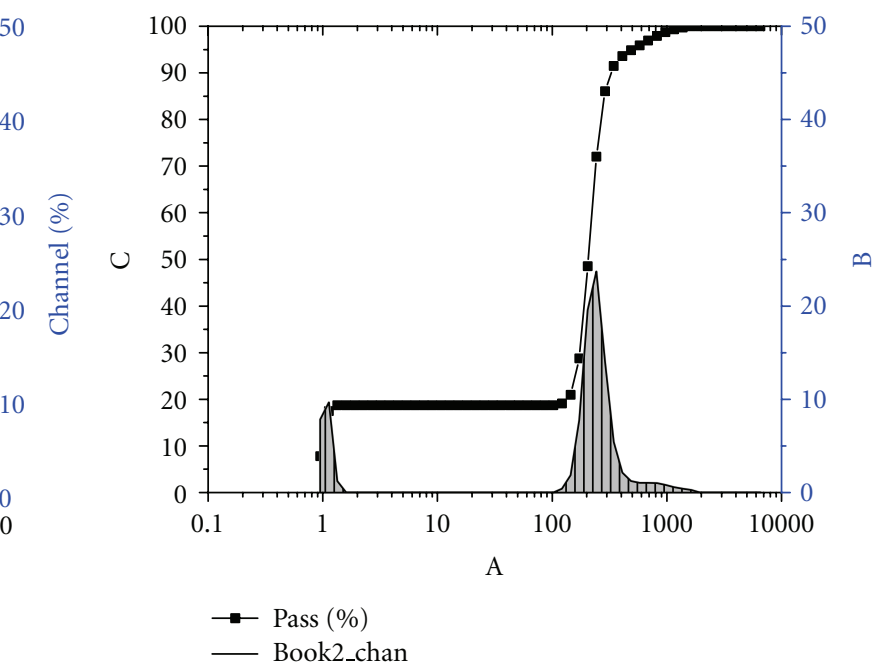

(f)

Figure 1: Particle size distribution of PLGA synthesized using (a) 1\% PVA Mol. Wt. 1, 15,000, (b) 1\% PVA Mol. Wt. 9000, (c) 0.5\%\% PVA Mol. Wt. 9000, (d) 1\% DMAB, (e) 0.5\% DMAB, and (f) 1\% SDS. 
TABLE 3: The berberine loading percentage onto PLGA nanoparticles prepared by different methods at pH 7.

\begin{tabular}{lcccccc}
\hline S.N. & Synthesis method & Solvent used for loading drug & Surfactant used & Berberine added & Particle size $(\mathrm{nm})$ & \% drug entrapment \\
\hline 1 & Single emulsion & Acetone & DMAB-0.3\% & $1 \mathrm{mg}$ & 1214 & 50 \\
2 & Single emulsion & Acetone & PVA-1\% & $2 \mathrm{mg}$ & 310 & 37.5 \\
3 & Single emulsion & DCM and acetone $(4: 1)$ & PVA-1\% & $3 \mathrm{mg}$ & 276 & 25 \\
4 & Double emulsion & DCM & PVA-1\% & $1 \mathrm{mg}$ & 182 & 30 \\
5 & Double emulsion & DCM and acetone $(4: 1)$ & PVA-1\% & $1 \mathrm{mg}$ & $298 \mathrm{~nm}$ & 40 \\
\hline
\end{tabular}

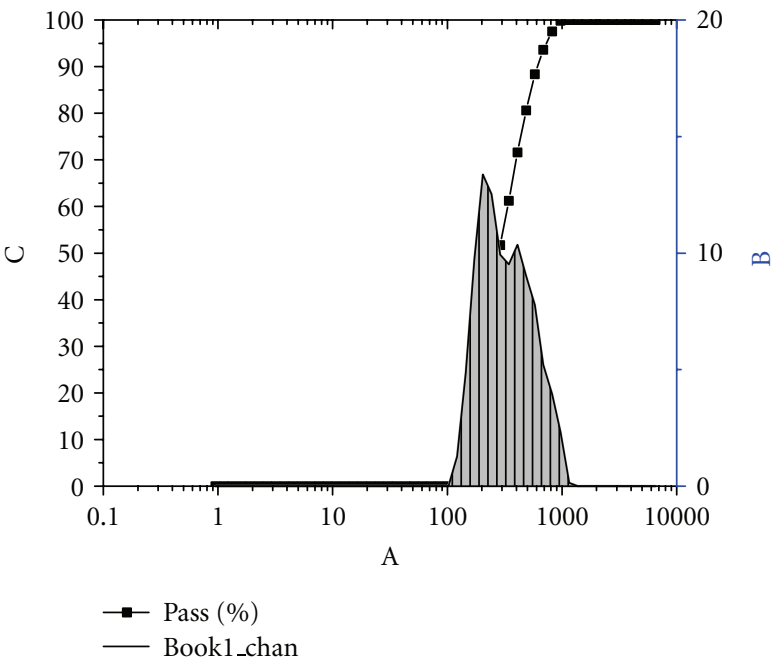

(a)

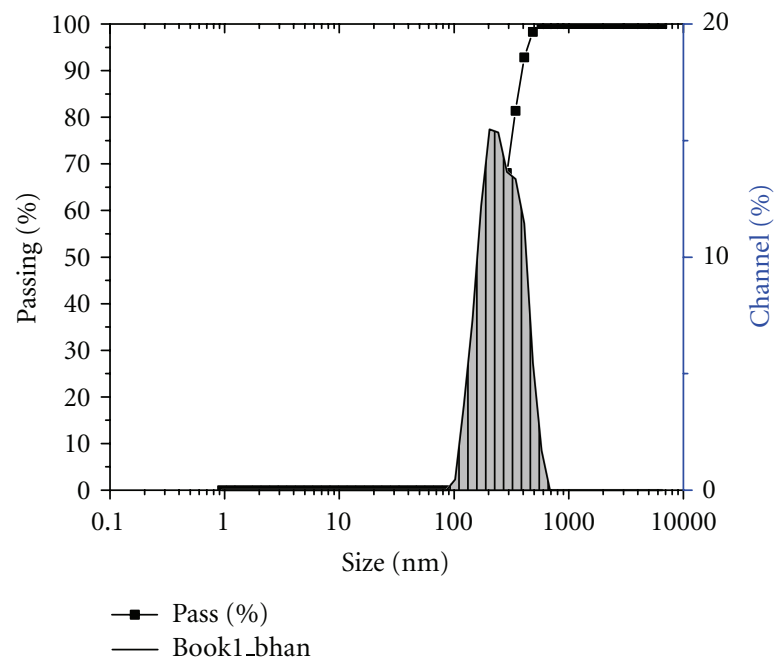

(b)

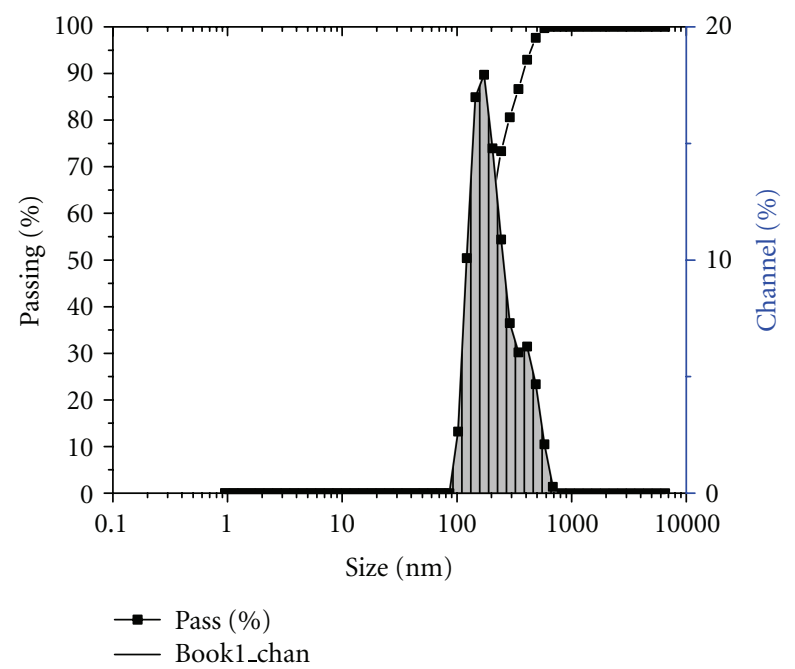

(c)

Figure 2: Particle size distribution of PLGA synthesized using 1\% PVA of Mol. Wt. 9000, along with solvents (a) acetone (b) EA, and (c) DCM.

drug in aqueous phase at basic pH (Table 4). The size of the berberine loaded PLGA nanoparticles varied from 150 to $200 \mathrm{~nm}$ (Figure 4(b)).

3.4. Particle Morphology. SEM analysis revealed that both PLGA NPs as well as berberine-loaded PLGA NPs were spherical in shape (Figure 4).
3.5. FTIR Analysis. Figure 5 exhibits the FT-IR spectra of, (a) berberine-loaded PLGA NPs, (b) PLGA and (c) Berberine. The broad strong peak at $3334 \mathrm{~cm}^{-1}$ of Figure 5(a) corresponds to $\mathrm{O}-\mathrm{H}$ stretching of adsorbed water in which the characteristic peaks of berberine in the range of 3200$3400 \mathrm{~cm}^{-1}$ (Figure 5(c)) has got merged. The strong characteristic peak at $1746 \mathrm{~cm}^{-1}$ in Figure 5(b) corresponds to 


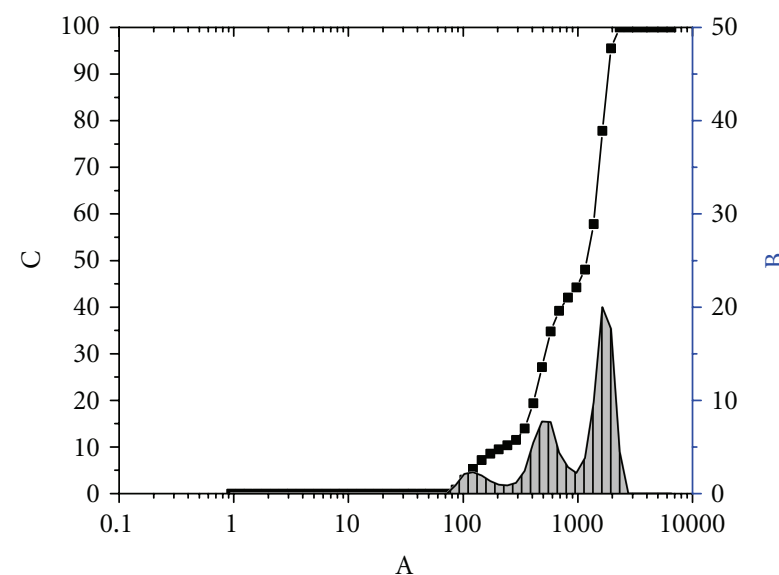

- Pass (\%)

_ Book1_chan

(a)

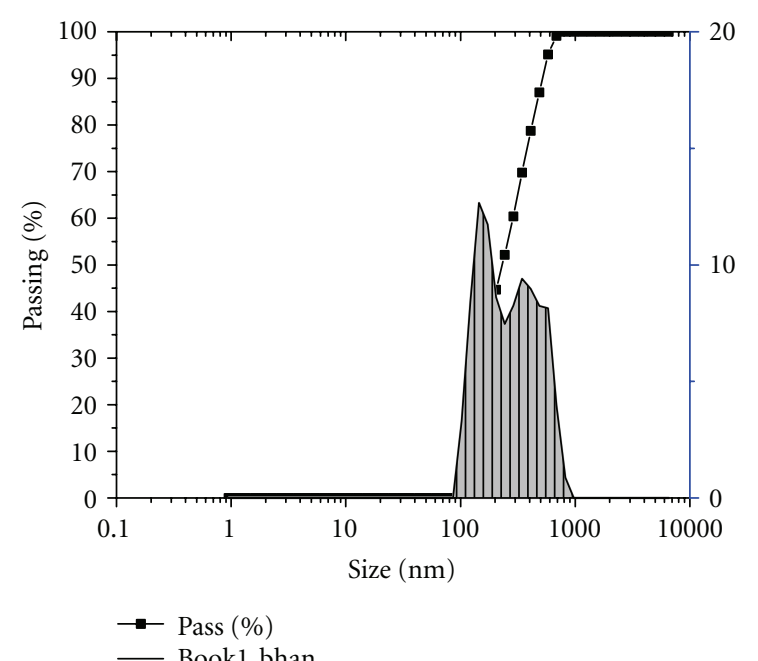

(c)

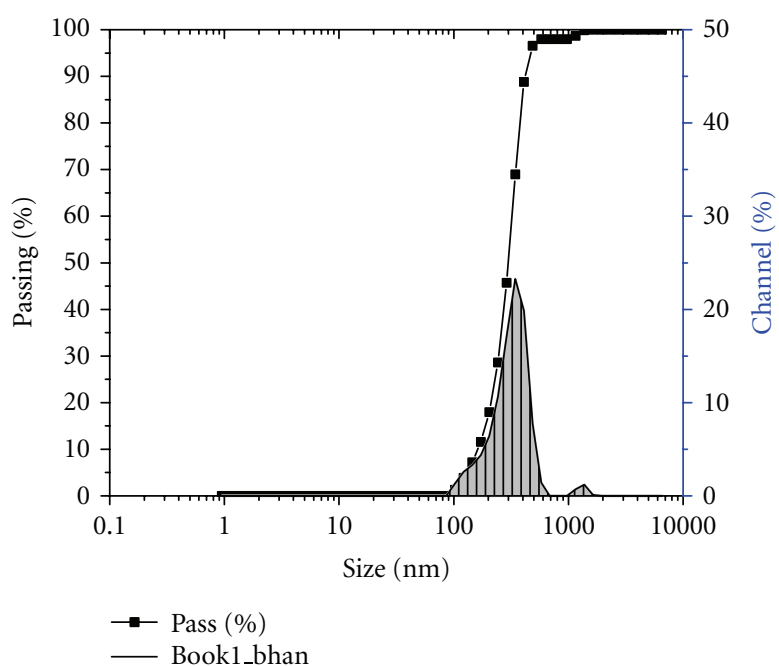

(b)

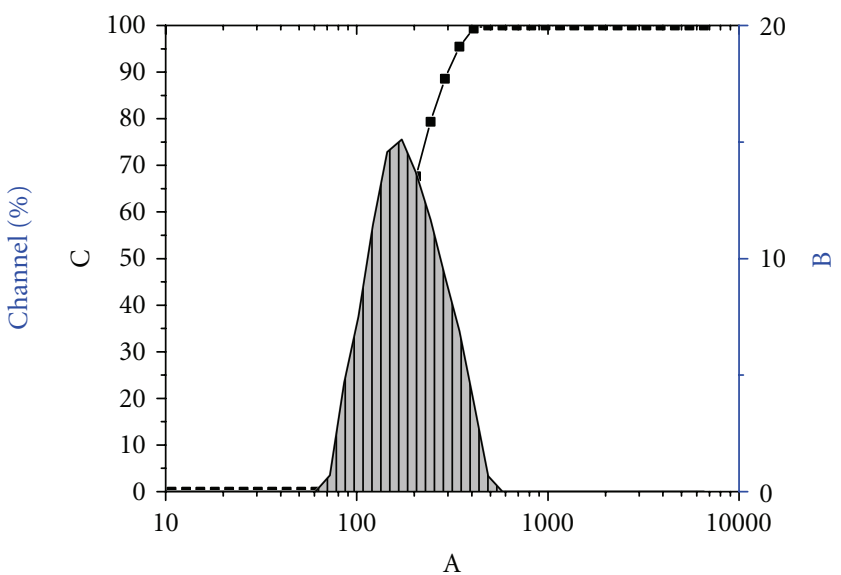

- Pass (\%)

_ Book1_chan

(d)

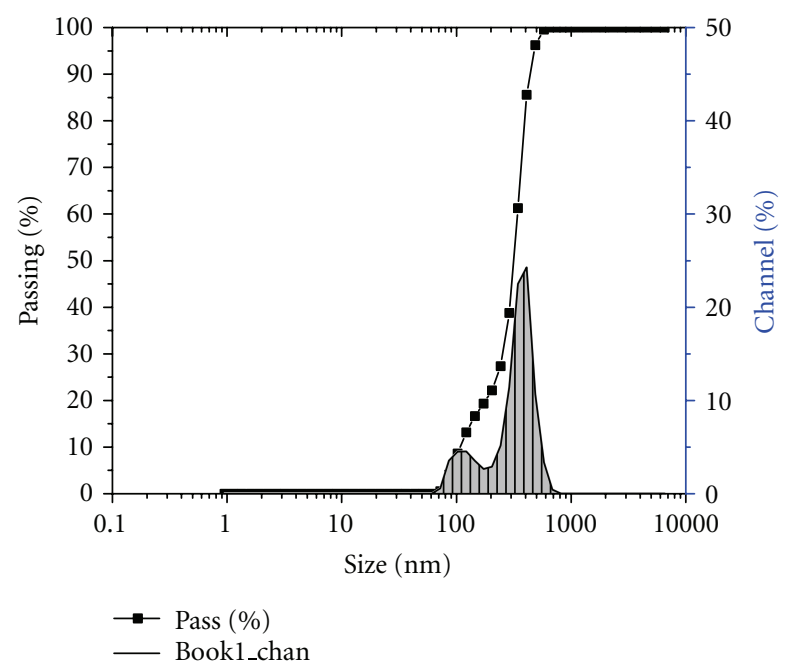

(e)

FIgURE 3: Particle size distribution of berberine + PLGA NPs prepared in (a) acetone + DMAB by single emulsion, (b) acetone + PVA by single emulsion, (c) acetone + DCM by single emulsion (d) DCM + PVA by double emulsion, (e) acetone + DCM by double emulsion. 


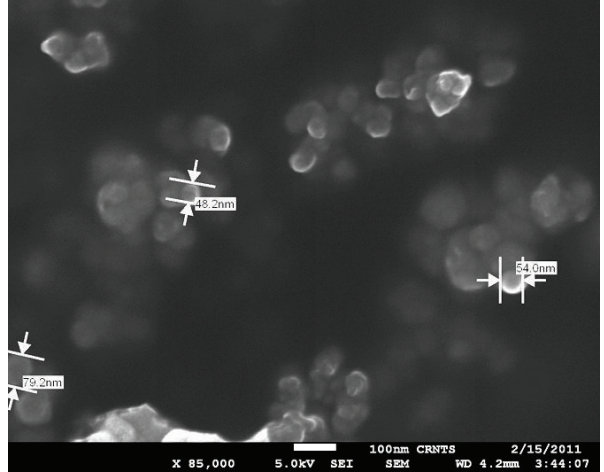

(a)

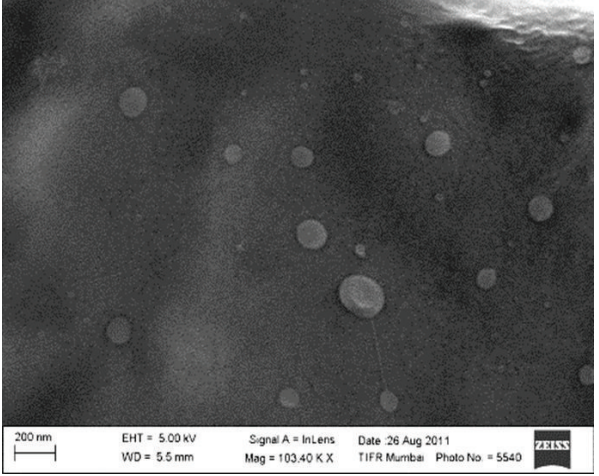

(b)

FIgURe 4: (a) PLGA NPs prepared using PVA and (b) Berberine loaded PLGA NPs.

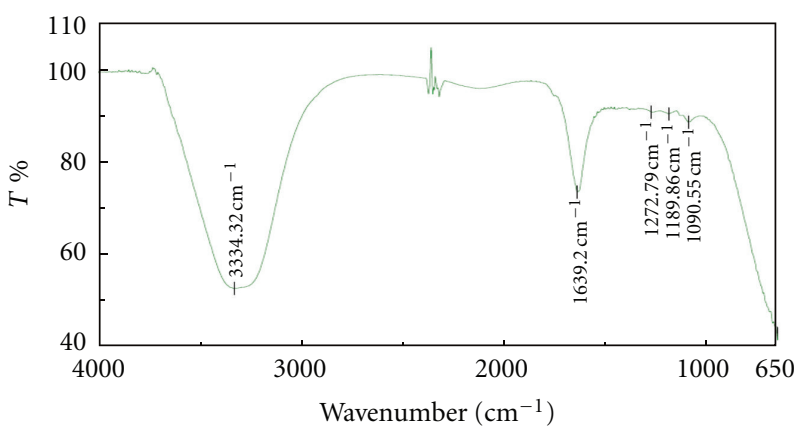

(a)

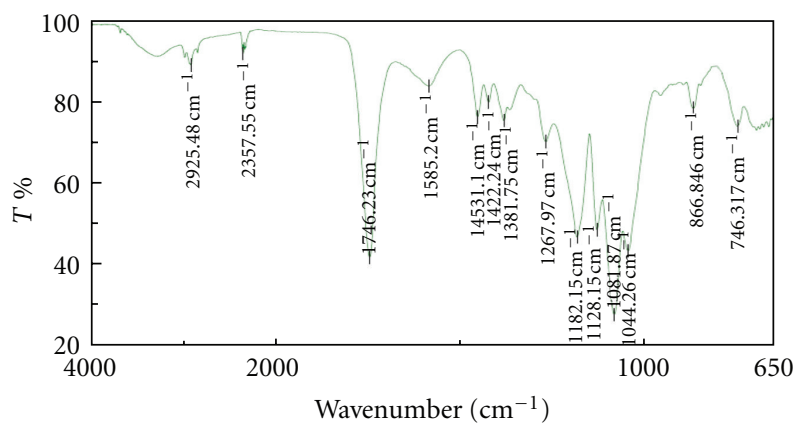

(b)

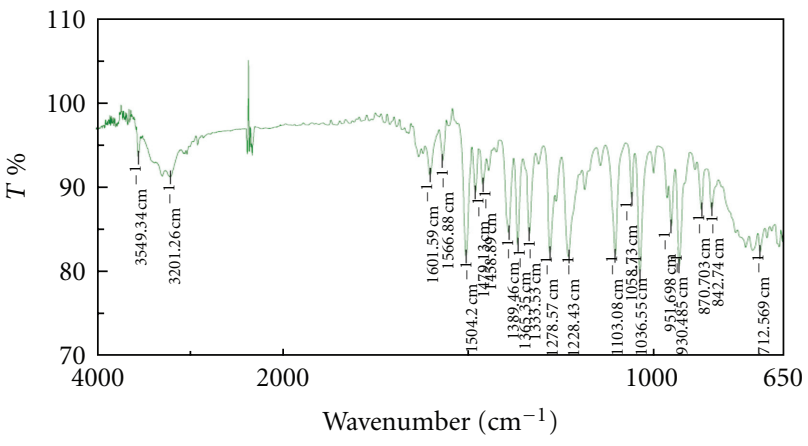

(c)

Figure 5: FT-IR spectra of (a) Berberine 1 + PLGA (b) 75: 25PLGA (c) Berberine.

TABLE 4: Effect of $\mathrm{pH}$ on the encapsulation efficiency of berberine in w/o/w method.

\begin{tabular}{lcccc}
\hline Sr. no. & $\mathrm{pH}$ & Solvent & Surfactant & $\%$ Loading \\
\hline 1 & 7 & DCM + Acetone $(4: 1)$ & PVA & $40 \%$ \\
2 & 8 & DCM + Acetone $(4: 1)$ & PVA & $58 \%$ \\
\hline
\end{tabular}

$\mathrm{C}=\mathrm{O}$ stretching of PLGA. This characteristic peak of carbonyl group is shifted to $1639 \mathrm{~cm}^{-1}$ (Figure 5(a)), which indicates chemical bonding between PLGA and berberine, may be through negatively charged carbonyl group of PLGA and positively charged nitrogen of berberine and also due to intermolecular hydrogen bonding with water molecules. Other characteristic peaks for $\mathrm{C}-\mathrm{O}$ and $\mathrm{C}-\mathrm{N}$ stretching of berberine can be seen in the range of $1090 \mathrm{~cm}^{-1}$ to $1272 \mathrm{~cm}^{-1}$ and other characteristic peaks of PLGA from 1100 to 1300 have also got merged into it.

3.6. Berberine Release Study. The in vitro release of berberine from PLGA was carried out in PBS at $\mathrm{pH} 5.5$ and 7.4 (Figure 6).

The drug release was faster at acidic $\mathrm{pH} 5.5$, almost 50\% drug was released in first 2 hours but afterwards the release was slow. A maximum of $70 \%$ drug was released in 24 hours, no more release was recorded after that (Figure 6). 


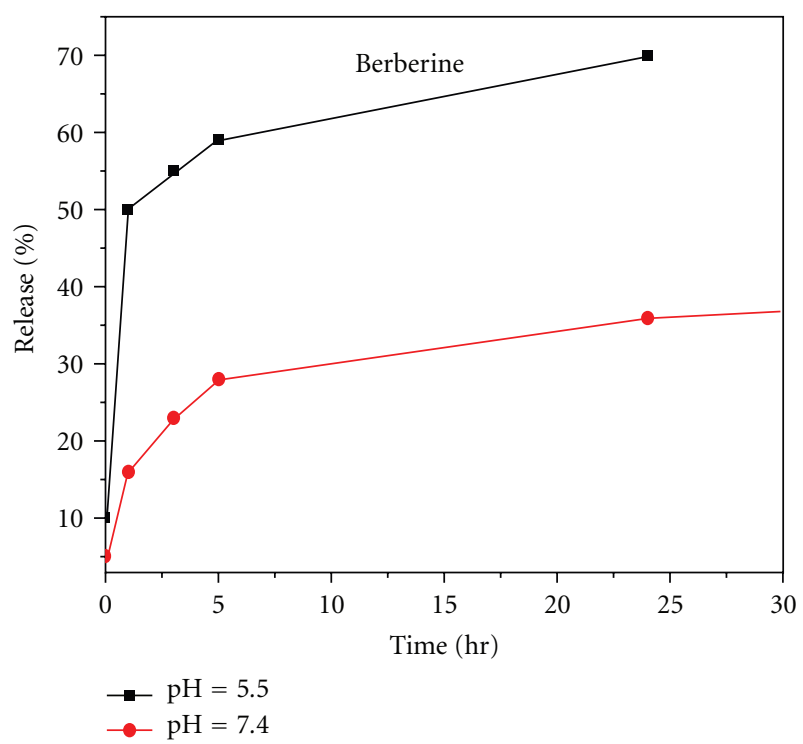

Figure 6: Release of berberine, encapsulated in PLGA NPs, at different $\mathrm{pH}$.

At $\mathrm{pH} 7.4$, the particles showed sustained drug release that is, only $16 \%$ drug was released in initial period of 2 hours and only $43 \%$ drug was released in 72 hours.

More release of drug in rather weak acidic medium may be because berberine is an alkaloid, which is basic. In strong acidic solution it may form salts and in near alkaline medium it will remain stably attached to PLGA. May be that is why better release was observed at $\mathrm{pH}$ 5.5.

Chang et al. [14] have tried heparin NPs as a vehicle for berberine and found that berberine conjugate can release the berberine in effectively controlled way at $37^{\circ} \mathrm{C}$. They used chitosan as a linker for preparing berberine/heparin/chitosan conjugate. Whereas Aihua et al. [15] used only chitosan NPs and loaded it with berberine to investigate the characterstics of in vitro release. Loading of berberine on chitosan was done by ionic gelation method. The NPs were dispersed with mean particle size of $267.9 \mathrm{~nm}$. the encapsulation was $65 \%$ out of which $56.8 \%$ drug was released within 6 hours in $0.9 \% \mathrm{NaCl}$. However in artificial gastric juice the release increased to $75.3 \%$. In PBS 7.4 only $45 \%$ berberine was released. Hence, they concluded that release is $\mathrm{pH}$ dependent. Another polymer tried for berberine encapsulation was calcium alginate [16] for releasing stomach specific drug.

\section{Conclusion}

The size of PLGA nanoparticles depends on factors like stabilizer and its concentration, type of organic solvent. By suitably selecting the various parameters like mode of homogenization, time of homogenization, stabilizer, and organic solvent, the NPs of desired size can be obtained.

Berberine-loaded PLGA NPs are successfully prepared using single emulsion as well as multiple emulsion method. The encapsulation efficiency was found to be more in multiple emulsion method. In case of single emulsion method the encapsulation efficiency decreased with the increase in concentration of drug for the same concentration of PLGA.

\author{
Abbreviations \\ DCM: Dichloromethane \\ DLS: Dynamic Light Scattering \\ DMAB: Didodecyl dimethyl ammonium \\ bromide \\ EA: $\quad$ Ethyl Acetate \\ NP: Nanoparticle \\ PBS: $\quad$ Phosphate buffer solution \\ PLGA: Poly (D, L-Lactide-co-glycolic acid) \\ PVA: Poly vinyl alcohol \\ SDS: $\quad$ Sodium dodecyl sulphate \\ DW: Distilled water.
}

\section{Acknowledgments}

Authors wish to acknowledge the support rendered by nsnRc and Nanotechnology Centre of University of Mumbai for particle size analysis. We are also indebted to UGC for providing financial assistance for the project. Our thanks are due, to Purasorb for supplying PLGA.

\section{References}

[1] L. F. Liu, "DNA topoisomerase poisons as antitumor drugs," Annual Review of Biochemistry, vol. 58, pp. 351-375, 1989.

[2] I. Bala, S. Hariharan, and M. N. V. R. Kumar, "PLGA nanoparticles in drug delivery: The state of the art," Critical Reviews in Therapeutic Drug Carrier Systems, vol. 21, no. 5, pp. 387-422, 2004.

[3] O. Kucuk, "Chemoprevention of prostate cancer," Cancer and Metastasis Reviews, vol. 21, no. 2, pp. 111-124, 2002.

[4] S. Barnes, "Role of phytochemicals in prevention and treatment of prostate cancer," Epidemiologic Reviews, vol. 23, no. 1, pp. 102-105, 2001.

[5] J. Cheng, B. A. Teply, I. Sherifi et al., "Formulation of functionalized PLGA-PEG nanoparticles for in vivo targeted drug delivery," Biomaterials, vol. 28, no. 5, pp. 869-876, 2007.

[6] G. V. Sathyavathi, A. K. Gupta, N. Tandon et al., Medicinal Plants of India, vol. 2, Indian Council of Medical Research, New Delhi, India, 1987.

[7] K. Fukuda, Y. Hibiya, M. Mutoh, M. Koshiji, S. Akao, and H. Fujiwara, "Inhibition of activator protein 1 activity by berberine in human hepatoma cells," Planta Medica, vol. 65, no. 4, pp. 381-383, 1999.

[8] J. G. Lin, J. G. Chung, and L. T. Wu, "Effects of berberine on arylamine Nacetyl-transferase activity in human colon tumor cells," The American Journal of Chinese Medicine, vol. 27, pp. 265-275, 1999.

[9] K. Fukuda, Y. Hibiya, M. Mutoh, M. Koshiji, S. Akao, and H. Fujiwara, "Inhibition by berberine of cyclooxygenase-2 transcriptional activity in human colon cancer cells," Journal of Ethnopharmacology, vol. 66, no. 2, pp. 227-233, 1999.

[10] S. Szeto, C. M. N. Yow, and K. W. Fung, "Characterization of berberine on human cancer cells in culture," Turkish Journal of Medical Sciences, vol. 32, no. 5, pp. 363-368, 2002.

[11] A. M. Roy, M. S. Baliga, C. A. Elmets, and S. K. Katiyar, "Grape seed proanthocyanidins induce apoptosis through p53, bax, 
and caspase 3 pathways," Neoplasia, vol. 7, no. 1, pp. 24-36, 2005.

[12] P. Ahlin, J. Kristl, A. Kristl, and F. Vrečer, "Investigation of polymeric nanoparticles as carriers of enalaprilat for oral administration," International Journal of Pharmaceutics, vol. 239, no. 1-2, pp. 113-120, 2002.

[13] R. Jalil and J. R. Nixon, "Biodegradable poly(lactic acid) and poly(lactide-co-glycolide) microcapsules: Problems associated with preparative techniques and release properties," Journal of Microencapsulation, vol. 7, no. 3, pp. 297-325, 1990.

[14] C. H. Chang, W. Y. Huang, C. H. Lai et al., "Development of novel nanoparticles shelled with heparin for berberine delivery to treat Helicobacter pylori," Acta Biomaterialia, vol. 7, no. 2, pp. 593-603, 2011.

[15] L. I. N. Aihua, L. I. Hongyan, L. I. U. Yiming, and Q. I. U. Xiaohui, "Preparation and release characteristics of berberine chitosan nanoparticles in vitro," China Pharmacy, 2007.

[16] Z.-H. Zhang, Y.-S. Sun, H. Pang, W. L. L. Munyendo, H.-X. Lv, and S.-L. Zhu, "Preparation and evaluation of berberine alginate beads for stomach-specific delivery," Molecules, vol. 16, no. 12, pp. 10347-10356, 2011. 

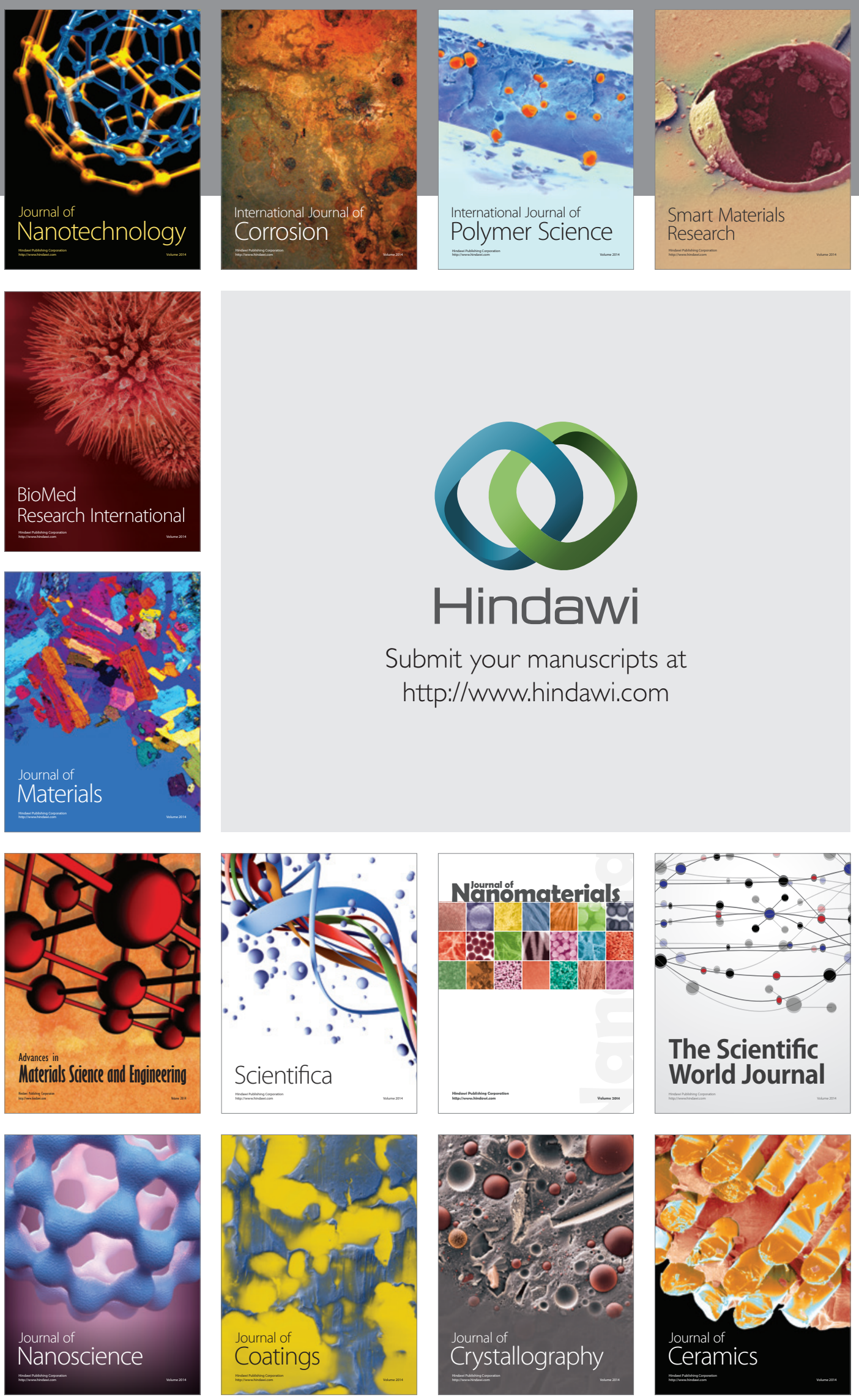

The Scientific World Journal

Submit your manuscripts at

http://www.hindawi.com

\section{World Journal}

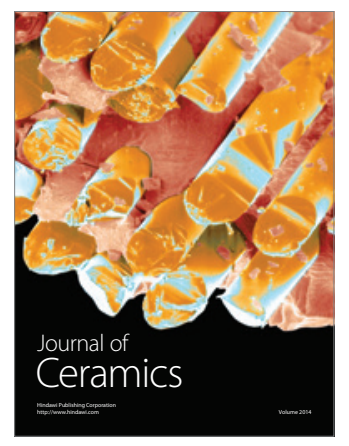

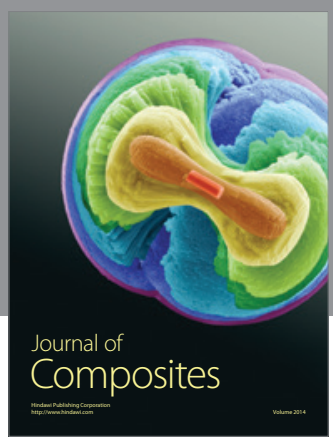
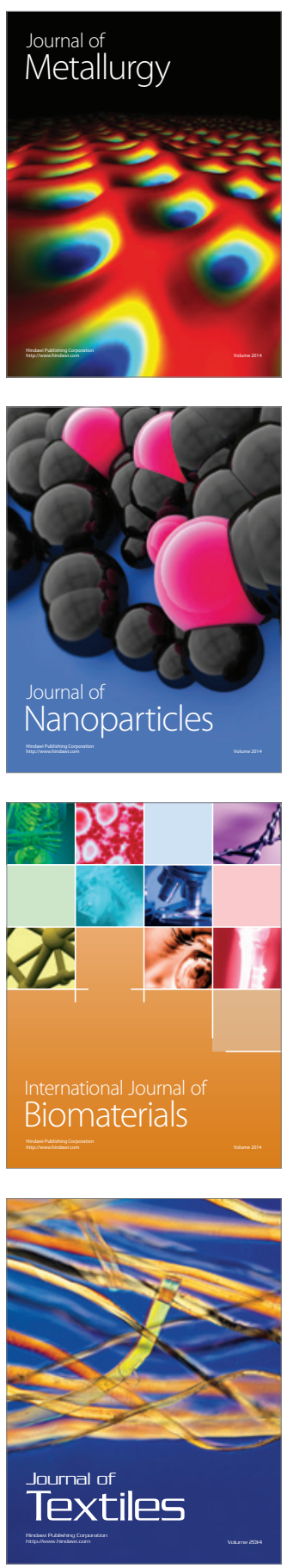